PDFlib PLOP: PDF Linearization, Optimization, Protection

Page inserted by evaluation version www.pdflib.com - sales@pdflib.com 


\title{
Human Rehearsal Processes and the Frontal Lobes: PET Evidence
}

\author{
EDWARD AWH, ${ }^{\circ}$ EDWARD E. SMITH, AND JOHN JONIDES \\ Department of Psychology \\ University of Michigan \\ Ann Arbor, Michigan 48109
}

\section{INTRODUCTION}

Our research is focused on working memory. This is a storage system that holds a limited amount of information for a brief time, with that information rapidly accessible and changeable from moment to moment. Such a system is essential for dealing with problems that require one to record features of a constantly changing environment, and to keep these features "on line" as they are used to guide behavior.

Extensive research supports two basic claims about the neural implementation of working memory in both human and nonhuman primates: (1) Working memory is implemented in the brain by heightened neural activity in particular regions, which notably include prefrontal and parietal cortex; $;^{1.2}$ and (2) different kinds of working memories correspond to different kinds of information. This is indicated by findings in nonhuman primates that neurons in different regions of the prefrontal cortex are active when monkeys have to store spatial information versus object information, ${ }^{3}$ and by the PET findings with humans that different networks of cortical regions are active depending on whether people have to remember spatial, object, or verbal information. ${ }^{4}$ In this paper, we emphasize human data, and try to go beyond the above two generalizations by decomposing each working memory into two basic constituents. We advance the following hypotheses:

1. Each working memory itself consists of a passive storage buffer and an active maintenance or rehearsal process. (The storage process may consist of either a separate structure or merely continued activation of perceptual processes; the rehearsal process consists of any operations that intentionally lengthen the duration of a representation.)

2. The storage and rehearsal processes may be implemented by different brain regions. In verbal working memory, rehearsal seems to be mediated by frontal regions, whereas storage may be mediated by posterior regions.

First we consider verbal working memory, and present evidence that it conforms to hypotheses 1 and 2. Then we consider spatial working memory and provide preliminary evidence characterizing the storage and rehearsal of information about location.

a Address correspondence to Edward Awh, 525 E. University Avenue, Ann Arbor, MI 48109-1109. 


\section{STORAGE AND REHEARSAL IN VERBAL WORKING MEMORY}

\section{Background}

The idea that verbal working memory includes both storage and rehearsal components is a cornerstone of behavioral research on working (or short-term) memory. ${ }^{5.6}$ One of the best pieces of evidence for the distinction is the word length effect. When a subject is presented a short list of words and asked to recall the words in order, accuracy is greater for words that take less time to pronounce. ${ }^{7}$ The simplest account of this finding is that subjects are rehearsing the words to themselves between presentation and recall, and the faster they can do so, the less likely that any particular word in store will decay before it is produced. A second piece of evidence for the storage-rehearsal distinction is the articulatory suppression effect. If subjects in a verbal working memory task are also required to articulate a familiar item concurrently (e.g., "the"), memory accuracy declines, and this decline cannot be attributed to simple distraction. ${ }^{6}$ The explanation of this result is that the concurrent articulation task disrupts or suppresses the subjects' implicit rehearsal, which in turn leads to decay of some of the items in store, and a decline in recall.

To follow up on this behavioral evidence, we conducted two PET experiments that investigate the storage-rehearsal distinction in verbal working memory. ${ }^{8}$

\section{Item Recognition Experiment}

\section{Rationale and Method}

In any verbal working memory task with a retention interval of a second or more, we would expect subjects to rehearse the material during the interval (as well as during the response period). ${ }^{9}$ Such rehearsal seems to consist of covert speech. Accordingly, it might involve the same neural mechanisms as does overt speech, which include Broca's area and other left-hemisphere frontal regions that appear to mediate the planning of speech. Thus, activation in these regions was expected in our task.

The first verbal working memory task that we studied was a variant of the widely used item recognition task developed by Sternberg. ${ }^{10}$ It is schematized at the top of Figure 1. Each trial consisted of four events: (1) a fixation cross presented for $500 \mathrm{~ms}$; (2) four targets, each one an uppercase letter, exposed for a total of $200 \mathrm{~ms}$; (3) a return to the fixation cross for a retention interval of 3000 $\mathrm{ms}$; and (4) a probe, available for $1500 \mathrm{~ms}$, that consisted of a single lowercase letter. The subjects' task was to decide whether or not the probe had the same name as any of the targets, and to indicate their decision by pressing a response button once for matches and twice for nonmatches. Because the targets and probe were in different cases, subjects were induced to represent the targets with a verbal code (phonological or articulatory). Successful performance on this task requires processes in addition to remembering verbal information, for example, attending to the inputs and selecting and executing a response. To remove the effects of these nonmemory processes from the PET images, a control condition 


\section{Item Recognition Task}

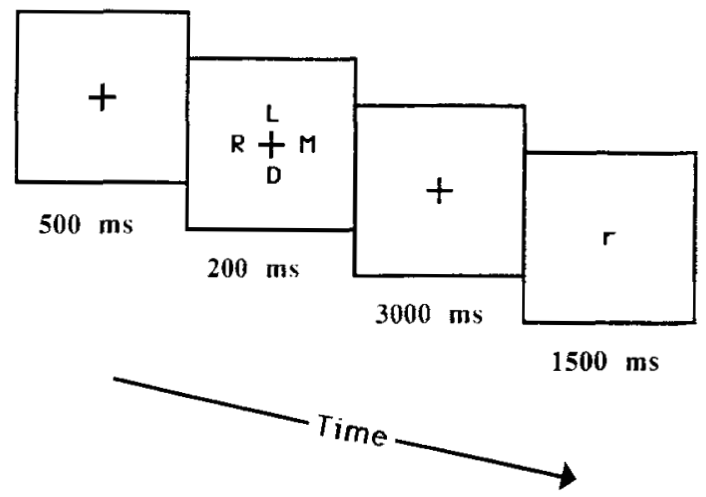

\section{Item Recognition Control}

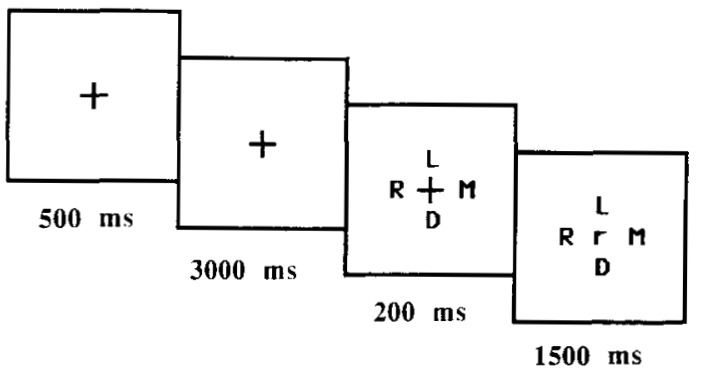

FIGURE 1. Schematic drawing of the events on each trial of the Item Recognition ( $t o p$ ) and Item Recognition Control (bottom) tasks.

was devised that included these processes but not memory (this follows the subtraction methodology developed by Posner et al. "). In the item recognition control task (FIG. I bottom), the sequence of trial events consisted of (1) a fixation cross; (2) continuation of the fixation cross for another $3000 \mathrm{~ms}$; (3) four uppercase target letters presented for a total of $200 \mathrm{~ms}$; and (4) a lowercase probe letter presented along with the four uppercase target letters. Again the subjects' task was to indicate whether or not the probe was identical in name to any of the targets, but in this case the decision could be made without any stored information. In both tasks, the probe matched one of the targets about half the time.

With regard to the PET procedure, subjects were first familiarized with the PET apparatus. Then each subject had an intravenous catheter inserted into his or her right arm for administration of the radioactive tracer. The subject was positioned in the scanner with a band across the forehead attached to a head 
holder to constrain head movement. Eleven subjects were tested. They had three scans each of the item recognition task and the control condition, in which each scan consisted of 20 trials in sequence. The sequence began just prior to the injection of the radionuclide, at which time a bolus injection of $66 \mathrm{mCi}$ of $\left[{ }^{15} \mathrm{O}\right]-$ labeled water was given. PET acquisition (using a Siemens/CTI-931/08-12) began $5 \mathrm{~s}$ after the count rate was observed to increase above the background level and continued for $60 \mathrm{~s}$ thereafter, during which time the sequence of items continued to be presented. The trials continued until after the PET scan was completed. Scans were performed at intervals of $14 \mathrm{~min}$, allowing time for the oxygen-15 to decay.

The PET images for each subject were first transformed to a stereotactic coordinate system,,$^{12.13}$ and then linearly scaled to the dimensions of a standard atlas brain. ${ }^{14}$ After normalizing pixel values for global flow-rate differences among scans, ${ }^{15}$ the data were averaged across subjects, thereby obtaining means and variances for the two conditions of interest. The difference image was created by subtracting activation in the control task from that in memory task; this image was then analyzed by performing post-hoc $t$ tests on a voxel-by-voxel basis and correcting the outcomes for multiple comparisons. ${ }^{16.17}$

\section{Results and Discussion}

The analysis resulted in a map of cerebral areas that showed significant increases in regional cerebral blood flow. FIGURE 2 presents four brain images showing the significant areas of activation in the item-recognition task. The number below each image gives its $z$-coordinate (an indication of how inferior or superior the relevant brain region is with respect to anterior-posterior commissure line). The areas of activation have been superimposed on a composite magnetic resonance image so as to provide some anatomical localization. The coordinates defining each area of activation are given in TABLE 1.

Note first that most of the significant areas of activation are in the left hemisphere. These regions include: Area 7 ("area" is shorthand for "Brodmann area") and area 40 in parietal cortex (see FIG. 2, top right image), along with three regions in frontal cortex, corresponding to area 44 (Broca's area), the inferior aspect of area 6 (the premotor area), and the superior aspect of area 6 (the supplementary motor area, or SMA). Broca's area is evident in the bottom left image, whereas the premotor area and SMA are visible in the top left image. The three left-hemisphere frontal regions are known to play a role in explicit speech, ${ }^{18}$ and hence are likely to mediate implicit speech, or rehearsal, as well. The two parietal regions may be involved in mediating the passive storage function. Indeed, area 40 in left parietal cortex is the most frequent site of damage in patients who have impaired verbal working memory. ${ }^{19.20}$

Other regions were activated as well. Two of these have surfaced in other PET studies of cognition: a right-hemisphere cerebellar site and a midline structure, the anterior cingulate. The cerebellar site has been argued to mediate aspects of the planning and execution of speech, ${ }^{21}$ so it too may play a role in implicit speech 


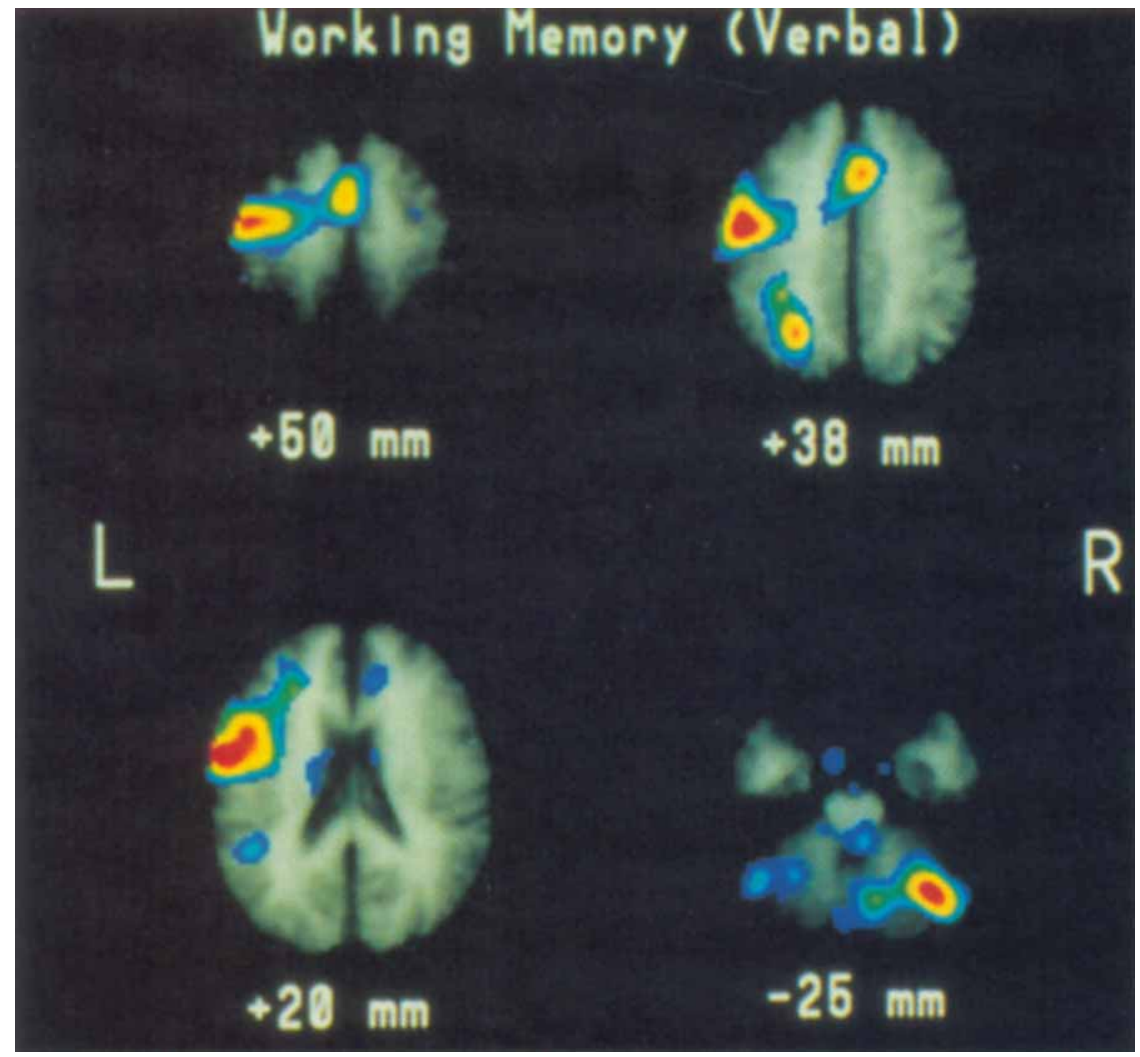

FIGURE 2. PET images of the statistically significant activation sites in the item Recognition minus Item Recognition Control subtraction analyses [Working Memory (Verbal)]. Each image is superimposed on an MRI image of a composite brain in order to illustrate anatomical localization of the activation foci. Stereotaxic coordinates of the significant foci of activation are given in TABLE 1 .

or rehearsal (the right cerebellum receives projections from the left cerebral hemisphere). The anterior cingulate has been found active in other studies of cognitive tasks, ${ }^{22}$ and has typically been interpreted as reflecting attentional processes. The remaining two areas of activation in the verbal task were the left-hemisphere thalamus and insular cortex (these are not shown in FiG. 2); we have no ready explanation for their involvement in the verbal task, nor are they typically found in other PET studies of working memory.

In sum, the known functionality of the activated areas supports the claim that different neuroanatomical regions mediate rehearsal and storage. In particular, whereas parietal regions may implement storage, frontal regions very likely implement rehearsal. Two of the frontal regions involved, the premotor area and SMA, 
Table 1. Significant Activation Foci for Memory Minus Control (Item Recognition Task)

\begin{tabular}{|c|c|c|c|c|}
\hline \multicolumn{3}{|c|}{ Stereotaxic Coordinates } & \multirow[b]{2}{*}{ Z-Score } & \multirow[b]{2}{*}{ Brain Area } \\
\hline $\mathrm{x}$ & $\mathrm{y}$ & $z$ & & \\
\hline & & & & Left hemisphere \\
\hline 24 & -55 & 43 & 5.3 & Posterior parietal (areas 40 and 7 ) \\
\hline 55 & 3 & 20 & 5.7 & Broca's area (area 44) \\
\hline 44 & 12 & 22 & 5.0 & Broca's area (area 44 ) \\
\hline 1 & 5 & 52 & 4.4 & SMA (area 6) \\
\hline 48 & -6 & 40 & 5.8 & Premotor cortex (area 6) \\
\hline 28 & 14 & 4 & 5.3 & Insular cortex \\
\hline 17 & -4 & 9 & 4.3 & Thalamus \\
\hline-33 & -60 & -25 & 5.4 & $\begin{array}{l}\text { Right hemisphere } \\
\text { Cerebellum }\end{array}$ \\
\hline & & & & Midline \\
\hline-6 & 19 & 38 & 4.6 & Anterior cingulate (area 32) \\
\hline
\end{tabular}

SMA, supplementary motor area.

are known to be involved in the high-level preparation and planning of movement in general for nonhuman primates as well as humans. ${ }^{23}$ It is no surprise, then, that these two areas also play a role in the planning of human speech. ${ }^{18}$ The other frontal region involved, Broca's area, is, of course, known to play a crucial role in overt speech; prior imaging work indicates it is also activated during internal speech, ${ }^{24}$ a result consistent with the present findings. Presumably, Broca's area mediates a more downstream function than that accomplished by the premotor area and SMA; for example, Broca's area may be responsible for specifying the articulatory features of the utterance to be internally generated.

\section{Continuous Memory Experiment}

\section{Rationale and Method}

One purpose of this study was simply to replicate the critical parietal (storage) and frontal (rehearsal) activations in a different kind of verbal working memory task. A second goal of this study was to provide more direct evidence that the frontal regions involved-Broca's, premotor, and SMA-mediate rehearsal. Our logic was similar to that used by Paulesu et $a l .^{21}$ These authors obtained PET measures during various tasks, which included an item recognition task similar to the one we used in the experiment just described and a rhyming task in which subjects had to decide whether or not each of a series of test letters rhymed with a target syllable. The item recognition task produced activations like those we obtained, including posterior parietal areas as well as frontal speech and motor sites. Assuming (as we do) that the item recognition task involved both rehearsal and storage, and assuming further that the rhyming task involved rehearsal but not storage, Paulesu et al. subtracted the activations in the rhyming task from those in the item recognition task. They found a reduction in the activation in the 
frontal regions but not in the parietal ones, and argued that this result supports the claim that the frontal regions mediate rehearsal. A problem with this logic, however, is that it is not clear that the rhyming task requires much by way of rehearsal-only one syllable needed to be maintained in the entire task. In lieu of this, we used as one of our conditions a task in which subjects were explicitly instructed to continuously rehearse letters. Presumably, this task reflects a purer measure of rehearsal, and subtracting its activation pattern from that obtained in our memory condition should come closer to "subtracting out rehearsal."

The verbal working memory task we used is referred to as "2-back" and is presented schematically at the top of FIGURE 3. Subjects were presented a continuous stream of single letters, each for $500 \mathrm{~ms}$ with a $2500-\mathrm{ms}$ interval between successive letters; each letter appeared at the center of the screen. The subjects' task was to decide whether or not each letter matched the one presented two back (not the previous letter, but the one prior to that). One-third of the letters provided matches that required positive responses (there were also three matches 1-back and 3-back so that subjects could not use mere familiarity as the basis of their responses).

There were two control conditions. One was a search task that presumably includes the nonmemory components of the 2-back task (e.g., encoding the letters, selecting and executing a motor response). This search condition is presented in the middle of Figure 3. The same sequence of letters as in the 2-back task was presented, but now subjects had only to decide whether or not each letter matched a target letter given at the beginning of the block. Subtracting the activation in this condition from that in the 2-back condition should reveal the neural bases of rehearsal and storage, which should match the results obtained in the previous study. The second control was a simple rehearsal task, and it is presented at the bottom of FigurF 3. Again the same sequence of letters as in the 2-back task was presented, but now subjects had to push a button when each letter appeared, continuously say the name of the letter to themselves until the next one appeared, and so on. Subtracting the activation in this condition from that in the 2-back condition should remove some of the neural underpinnings of rehearsal, but leave the neural basis of storage unaffected.

The PET procedure was the same as that in the previous experiment, except that now nine subjects were tested, and every subject had three scans of each of the three conditions. The analysis of the PET images paralleled that in the first experiment.

\section{Results and Discussion}

We are again interested in difference images, but now two subtractions are of particular interest: 2-back minus search and 2-back minus rehearsal. FIGURE 4 presents the difference images for 2-back minus search: the coordinates defining each significant area of activation are given in the top of TABLE 2.

The most important result is that all areas that were significantly activated in the first study are again significant here, with the exception of the two regions for which interpretation was unclear: thalamus and insular cortex. Again, there is 

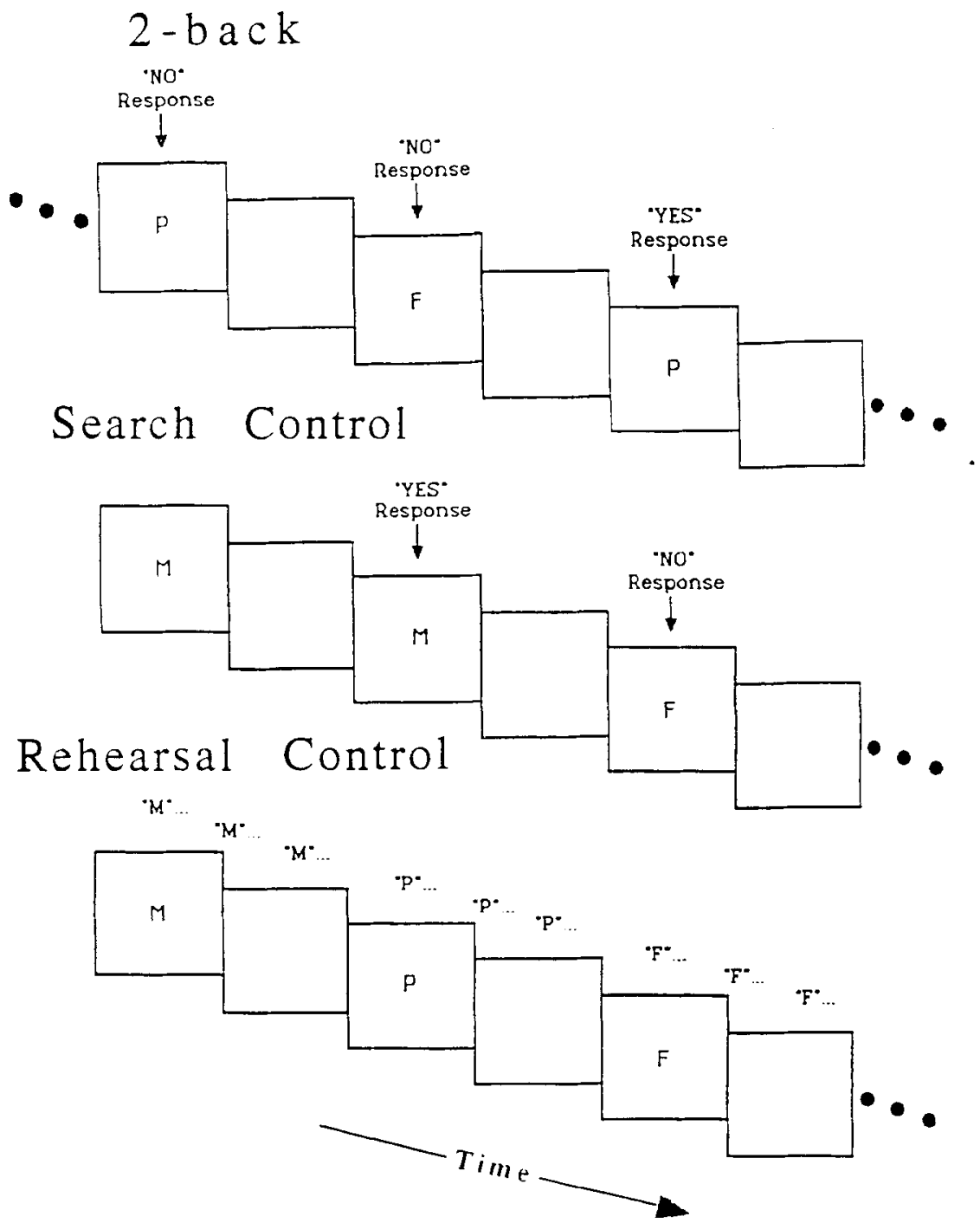

FIGURE 3. Schematic drawing of the sequence of events and the appropriate response for each item in a sample series from the 2-Back (top), Search (middle), and Rehearsal (bottom) conditions. 
TABle 2. Significant Activation Foci for 2-Back Minus Search

\begin{tabular}{rrrrl}
\hline \multicolumn{2}{c}{ Stereotaxic Coordinates } & & \\
\hline $\mathrm{x}$ & $\mathrm{y}$ & $\mathrm{z}$ & Z-Score & \multicolumn{1}{c}{ Brain Area } \\
\hline & & & Left hemisphere \\
33 & -46 & 38 & 5.4 & Posterior parietal (area 40) \\
17 & -60 & 45 & 5.7 & Superior parietal (area 7) \\
42 & 17 & 22 & 4.4 & Broca's area (area 44) \\
28 & 1 & 52 & 6.0 & SMA, premotor (area 6) \\
26 & -67 & -50 & 5.1 & Cerebellum \\
6 & 3 & 54 & 5.2 & SMA \\
& & & & Right hemisphere \\
-12 & -64 & 47 & 5.3 & Superior parietal (area 7) \\
-26 & -55 & 50 & 4.6 & Superior parietal (area 7) \\
-24 & 3 & 52 & 5.5 & SMA, premotor (area 6) \\
-1 & -64 & -25 & 4.8 & Cerebellar vermis \\
-33 & -60 & -25 & 5.4 & Cerebellum \\
& & & & Midline \\
3 & 12 & 40 & 5.0 & Anterior cingulate (area 32) \\
\hline
\end{tabular}

SMA, supplementary motor area.

activation in the three frontal regions that presumably mediate rehearsal-Broca's area, premotor area, and SMA - as well as in the posterior parietal regions that presumably implement storage. Furthermore, the areas common to our two studies have also been found in studies of other investigators of verbal working memory. ${ }^{21.25}$ This convergence of results strengthens our belief that we are indeed seeing evidence of the neural basis of verbal working memory.

In addition to these common areas of activation, three additional areas proved significant in the 2-back minus search subtraction. All three are right-hemisphere regions homologous to those activated in the left hemisphere, and include Area 7 in the superior parietal cortex, along with the premotor area and SMA in frontal cortex. The degree of activation in these right-hemisphere areas is consistently less than that in the left-hemisphere homologues. These findings suggest that the right-hemisphere activations under discussion may reflect the functional recruitment of right-hemisphere mechanisms to assist in an unusually demanding version of what is normally a left-hemisphere task. ${ }^{26}$

Consider now the 2-back minus rehearsal subtraction, which should reveal activations in brain areas that mediate storage with areas related to rehearsal subtracted out. FiGURE 5 presents the difference images; the coordinates defining each significant area are given in TABLE 3. As expected, this subtraction reveals a loss of significant activation in Broca's area and premotor area, both of which presumably mediate rehearsal. Furthermore, the activation in left posterior parietal cortex remains significant, supporting the hypothesis that this area participates in storage. However, there is still activation in SMA and right cerebellar cortex after subtraction, despite the fact that these areas have also been associated with rehearsal. One possibility is that our rehearsal control was not sufficiently demanding to engage a full complement of rehearsal processes. Alternatively, these areas may mediate processes unrelated to rehearsal.

The 2-back minus rehearsal subtraction also reveals activation in the thalamus, 


\section{Back - Search}
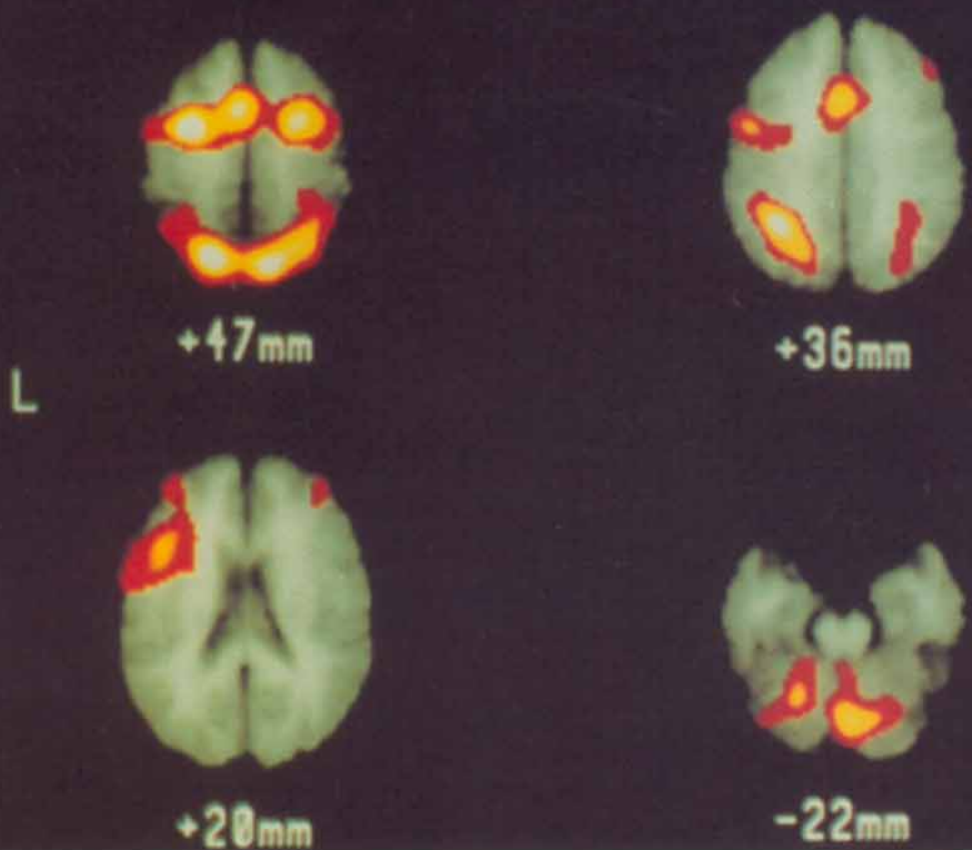

R

\section{Back - Rehearıal}
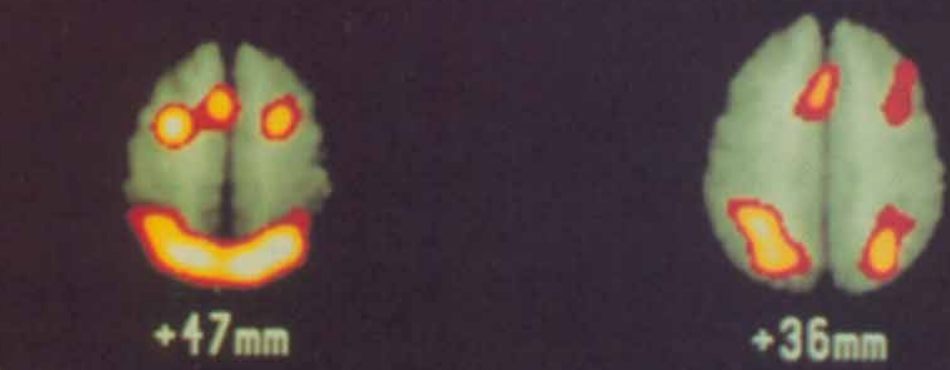

L

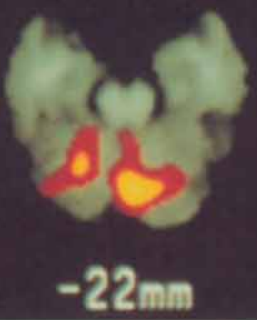

$+36 \mathrm{~mm}$

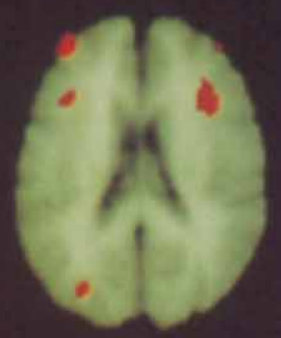

$+28 \mathrm{~mm}$

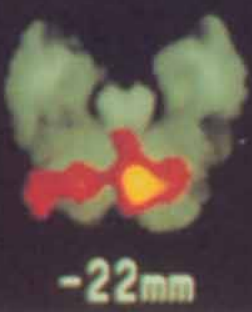


TABLE 3. Significant Activation Foci for 2-Back Minus Rehearsal

\begin{tabular}{|c|c|c|c|c|}
\hline \multicolumn{3}{|c|}{ Stereotaxic Coordinates } & \multirow[b]{2}{*}{ Z-Score } & \multirow[b]{2}{*}{ Brain Area } \\
\hline $\mathrm{x}$ & $y$ & $\mathrm{z}$ & & \\
\hline 17 & -60 & 43 & 5.8 & $\begin{array}{l}\text { Left hemisphere } \\
\text { Posterior and superior parietal } \\
\text { (areas } 40 \text { and } 7 \text { ) }\end{array}$ \\
\hline 28 & 1 & 50 & 5.5 & SMA \\
\hline 3 & 14 & 43 & 4.8 & SMA \\
\hline $\begin{array}{r}-26 \\
-12 \\
-26 \\
-3 \\
-3 \\
-28\end{array}$ & $\begin{array}{r}-58 \\
-64 \\
3 \\
-17 \\
-62 \\
-60\end{array}$ & $\begin{array}{r}45 \\
47 \\
50 \\
2 \\
-25 \\
-38\end{array}$ & $\begin{array}{l}5.3 \\
5.7 \\
4.5 \\
4.7 \\
4.8 \\
4.6\end{array}$ & $\begin{array}{l}\text { Right hemisphere } \\
\text { Superior parietal (area 7) } \\
\text { Superior parietal (area 7) } \\
\text { SMA (area 6) } \\
\text { Thalamus } \\
\text { Cerebellar vermis } \\
\text { Cerebellum }\end{array}$ \\
\hline
\end{tabular}

SMA, supplementary motor area.

for which we have no interpretation. Finally, the activation in anterior cingulate cortex that appeared in the 2-back minus search subtraction drops out in this subtraction. The lack of anterior-cingulate activation casts doubt on interpretations that center on an attentional role for this brain region because the attentional difference between 2-back and rehearsal seem similar to that between 2-back and the search control.

In sum, these results provide two sources of evidence for the claim that verbal rehearsal is mediated by regions in frontal cortex. First, using a very different working memory task than that employed in the first study, we again found significant activation in Broca's area, premotor area, and SMA-regions whose known functionality involves the planning of speech. Second, we also provided "subtrac. tion" evidence for the claim of interest. We selected two tasks-2-back and rehearsal-which at the cognitive level seem to require implicit rehearsal, and then showed at the neural level that the active regions they have in common tend to be those that mediate implicit rehearsal.

A weak link in the above argument is the assumption that, at the cognitive level, both the 2-back and rehearsal tasks require rehearsal. Such an assumption calls for behavioral evidence. Accordingly, we performed a follow-up behavioral experiment in which we sought to establish an articulatory suppression effect, which is a behavioral indicator of rehearsal (see our earlier discussion). Subjects

FIGURE 4 (Top). PET images of the statistically significant activation sites in the 2-Back minus Search subtraction analyses. Each image is superimposed on an MRI image of a composite brain. Stereotaxic coordinates of the significant foci of activation are given in TABLE 2.

FIGURE 5 (Bottom). PET images of the statistically significant activation sites in the 2-Back minus Rehearsal subtraction analyses. Each image is superimposed on an MRI image of a composite brain. Stereotaxic coordinates of the significant foci of activation are given in TABLE 3. Note the loss of significant activation in anterior regions associated with rehearsal (i.e., Broca's area and premotor area), whereas left posterior parietal activations remain significant. 


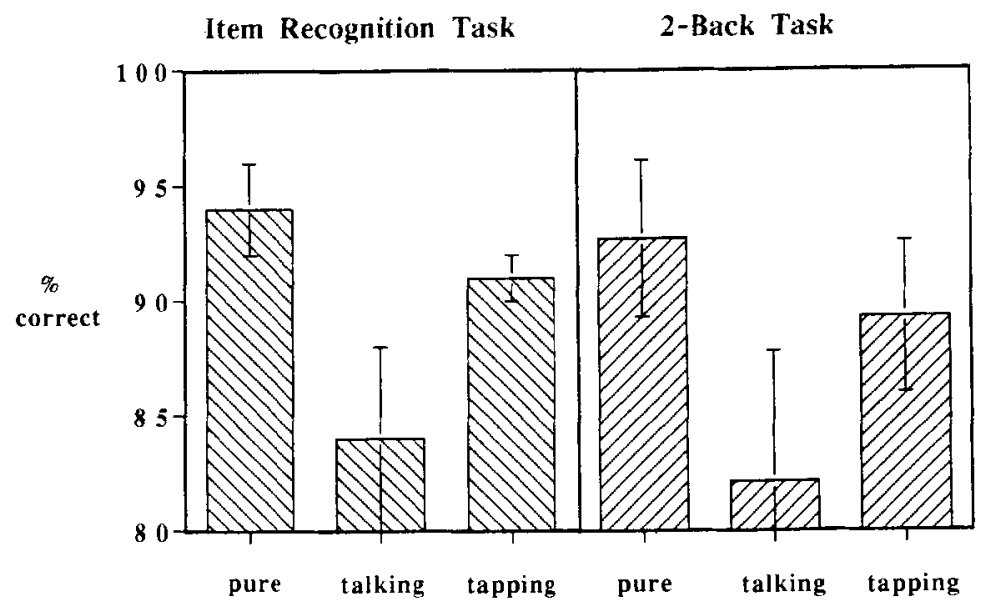

FIGURE 6. Graphs depicting Item Recognition (left) and 2-Back (right) accuracies during pure task performance, articulatory suppression, and tapping. In both tasks, only articulatory suppression caused significant decrements in performance. Because two-thirds of the 2-back trials required negative responses, there could have been an overall bias to say "no": hence, only the data from match trials are presented (this pattern of accuracies is also statistically significant across all trials).

performed the 2-back task or the item recognition task from the first study, either alone or concurrently with another task. One concurrent task required subjects to continuously say aloud the names of the digits $1-4$, whereas the other secondary task required subjects to continuously tap with the fingers of the left hand at a prescribed rate; only the "say aloud" or articulation task should interfere with implicit rehearsal, and hence this task should be more detrimental to memory performance than the tapping task. Exactly this difference was found in both the 2-back and the item recognition tasks (see FiG. 6). Thus the behavioral results converge with the PET findings in indicating that rehearsal is involved in the memory tasks that we studied.

\section{Summary}

Our PET results are broadly consistent with the generalization that part of the neural network mediating working memory is in the frontal cortex. ${ }^{1}$ However, in our studies the frontal cortex contribution to working memory involves rehearsal not sheer storage. Of course, the results discussed thus far concern only verbal materials; whether our notion of rehearsal processes applies to nonverbal working memory is a topic which we next address.

\section{REHEARSAL IN SPATIAL WORKING MEMORY}

\section{Previous Hypotheses}

Research on spatial working memory has focused mainly on its independence from other working memory systems. There have been few hypotheses regarding 
the specific subcomponents of this system. Baddeley ${ }^{6}$ has suggested that implicit eye movements (i.e., operation of an eye-movement control system without overt eye movements) to target positions may serve as a rehearsal mechanism for spatial positions. He reports that a secondary task requiring overt eye movements disrupts the performance of a spatial memory span task. Smyth and Scholey ${ }^{27}$ hypothesize that spatial working memory depends on shifts of spatial attention. They demonstrated that spatial span (assessed by a version of the Corsi Blocks task) is reduced by concurrent tasks that require shifts of spatial attention. It is still unclear, however, exactly how spatial attention might mediate the rehearsal of location information. In the following sections, we provide a hypothesis that makes explicit the role of spatial selective attention.

\section{A Specific Hypothesis}

Research on spatial selective attention emphasizes improvements in processing efficiency at attended locations in space. ${ }^{28}$ Thus, although it is well known that stimuli falling in the center of the visual field enjoy faster and more sensitive perceptual processing (the retinal acuity effect), visual processing advantages independent of this factor can be demonstrated when attention is oriented to a particular region of space without shifts of gaze. Current research suggests that spatial selective attention may operate by enhancing the processing of early perceptual systems in a location-specific manner. ${ }^{29}$ Thus, visual processing may be improved in the particular cortical regions that process the attended region of space. (Recall that visual cortex is topographically organized-that is, independent locations in cortex can be mapped onto independent locations in the environment.)

We propose a model of spatial rehearsal in which an interaction of attentional and perceptual mechanisms mediates the "on line" maintenance of spatial information. By this account, the rehearsal of spatial information corresponds to spatial selective attention, creating a location-specific change in visual processing mechanisms. Two forms of support for this view are offered: First, a review of evidence suggests a strong correspondence between the neuroanatomical regions mediating spatial selective attention and those that we have found to mediate spatial working memory. Second, a preliminary behavioral study provides evidence of interaction between the psychological mechanisms of spatial selective attention and spatial working memory.

\section{Brain Circuitry Involved in Spatial Selective Attention}

\section{Evidence for Enhancement of Early Visual Processing}

A recent study by Heinze et $a .^{29}$ supports the idea that visual processing in extrastriate cortex is modulated by spatial selective attention. Subjects in these experiments were instructed to attend to either the left or right side of bilateral stimulus arrays to perform a visual target detection task. Combined PET and ERP measures showed increased activation in extrastriate cortex (contralateral to the 
attended side) that began as early as $80-130 \mathrm{~ms}$ after stimulus onset. Thus, both the timing and localization of these neuronal responses suggest that spatial selective attention causes location-specific changes in early visual processing.

\section{Parietal Cortex}

The neural network mediating spatial selective attention has also been shown to include regions of parietal cortex ${ }^{30}$ When attention was tonically maintained to a specific spatial location, increased activation occurred in parietal-occipital cortex (once again, contralateral to the attended side of space). In addition, similar contralateral increases were observed in the inferior occipital association cortex. The interpretation of these results was that the visual responses in these areas were enhanced by orienting of selective attention. Furthermore, in a task where subjects continuously shifted attention to various peripheral locations, enhanced responses were observed in superior parietal cortex.

\section{Frontal Cortex}

In a detection task, the activity of neurons in the monkey prefrontal cortex was recorded. ${ }^{31}$ Visual stimuli were presented extrafoveally while the monkey gazed at a fixation point. In one condition, the monkey was attending the peripheral visual stimulus (because the behavioral response depended on the offset of this stimulus). In another condition, the peripheral stimulus did not have behavioral significance. When neurons in the prearcuate and periprincipalis areas (putatively homologous to dorsolateral prefrontal cortex in humans ${ }^{32}$ ) were compared in these two conditions, significantly higher neuronal responses were found to attended visual stimuli. These results provide support for a role of prefrontal cortex in the selection of visual stimuli.

It should be noted that frontal lobe regions were also activated in the studies by Heinze et al..$^{29}$ and Petersen et al. ${ }^{30}$ In particular, both studies showed that area 6 (including premotor and supplementary motor areas) is activated by orienting of spatial attention. Further evidence comes from a recent review of the effects of frontal lobe damage in humans. ${ }^{33}$ The authors concluded that regions of frontal cortex are critical to the proper functioning of spatial selective attention. For example, a study of patients who had undergone unilateral frontal or temporal lobe excisions ${ }^{34}$ revealed that frontal lobe patients were less able to take advantage of a cue that indicated the location of an impending visual target. From this and other studies, Foster et al. concluded that the right frontal lobe is particularly important for visuospatial orienting.

\section{Summary of Spatial Selective Attention Circtitry}

The following cortical regions have been implicated in spatial selective attention: (1) visual processing areas, including extrastriate and parietal-occipital regions; (2) superior parietal cortex; and (3) frontal cortex, including dorsolateral 
prefrontal cortex and area 6 (supplementary motor and premotor). Next, we consider the overlap between these regions and those that mediate spatial working memory.

\section{Spatial Working Memory Experiment}

\section{Method}

The spatial working memory task we used is referred to as "spatial 3-back" and is illustrated in Figure 7. This task is very similar to the continuous verbal memory experiment described previously. Subjects fixed their gaze on a centrally located cross while a sequence of 42 consonants was presented, each for 500 $\mathrm{ms}$, with a 2500 -ms interval between successive letters. The letters appeared at randomly chosen locations around an imaginary circle (whose radius was $6.6^{\circ}$ of visual angle). Their task was to decide whether the spatial position of each letter matched the position of the letter presented three back. This spatial memory experiment was part of a larger study that also tested verbal working memory; however, only the spatial working-memory results will be discussed here.

The control condition was a spatial search task designed to include the perceptual and motor components of the spatial 3-back task. The stimulus display for the search task was identical to the one used in the spatial 3-back task, but the subjects' task here was simply to search for the occurrence of one of three previously memorized spatial positions. Subtracting the activation in this spatial search task from that in the spatial 3-back task should reveal the brain regions that mediate storage and rehearsal of spatial information.

Again, the PET procedure was identical to that used in the continuous verbal memory study, except that eight subjects were tested; each subject had two scans of the spatial 3-back condition, and two scans of the search condition. The analysis

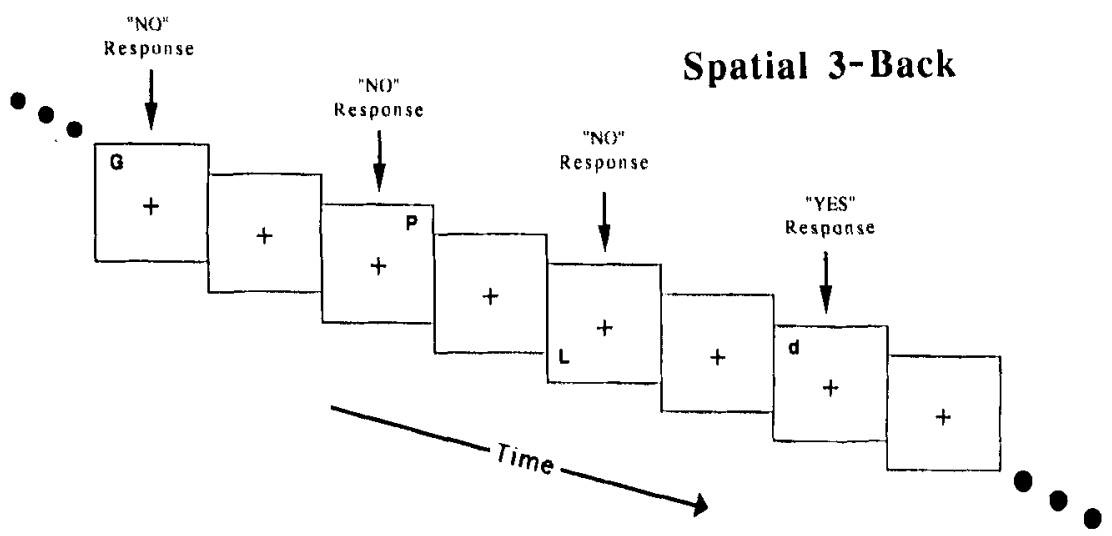

FIGURE 7. Schematic drawing of the sequence of events and the appropriate response for each item in a sample series from the Spatial 3-Back condition. 
techniques on the difference images are consistent with the other PET experiments in this report.

\section{Results and Discussion}

The difference images for spatial 3-back minus spatial search are presented in Figure 8 . The coordinates and $z$-score for each activation focus are shown in TABLE 4.

The most striking feature of these results is the strong overlap with the neural circuitry associated with spatial selective attention. Specifically, the subtraction analyses reveal a bilateral pattern of activation in superior and inferior parietal cortex (areas 7 and 40), dorsolateral prefrontal cortex (areas 9, 10, and 46), and supplementary motor cortex (area 6). All of these sites have been implicated in the neural circuitry underlying spatial selective attention. Moreover, the frontal and posterior parietal activations show a clear right-hemisphere dominance (as indicated by the z-scores for the right- and left-hemisphere sites), consistent with neuropsychological evidence on spatial attention. ${ }^{3.3}$

The extrastriate region is the only cortical area implicated in the selective attention studies that was not found in the present analysis. However, there is reason to believe that the particular control task we used may have caused this anomaly. Recall that subjects were searching for the occurrence of any of three

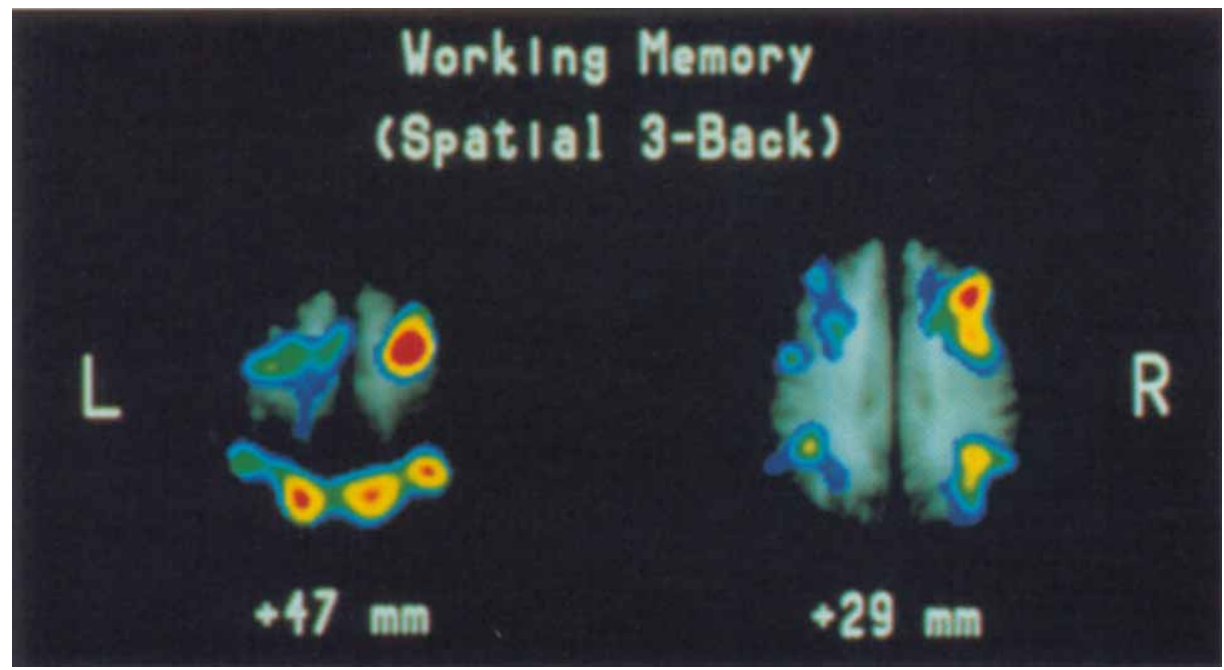

FIGURE 8. PET images of the statistically significant activation sites in the Spatial 3-Back ininus Search subtraction analyses. Each image is superimposed on an MRI image of a composite brain. Stereotaxic coordinates of the significant foci of activation are given in TABLE 4 . Note the relative dominance of right-hemisphere activation in this task. 
TABLE 4. Significant Activation Foci for Spatial 3-Back Minus Spatial Search

\begin{tabular}{|c|c|c|c|c|}
\hline \multicolumn{3}{|c|}{ Stereotaxic Coordinates } & \multirow[b]{2}{*}{ Z-Score } & \multirow[b]{2}{*}{ Brain Area } \\
\hline $\mathrm{x}$ & $\mathrm{y}$ & $\mathrm{z}$ & & \\
\hline & & & & Left hemisphere \\
\hline 44 & -46 & 43 & 5.5 & Posterior parietal (area 40) \\
\hline 19 & -67 & 50 & 5.0 & Superior parietal (area 7) \\
\hline 26 & -53 & 40 & 4.8 & $\begin{array}{l}\text { Posterior and superior parieta } \\
\text { (areas } 40 \text { and } 7)\end{array}$ \\
\hline & 44 & 20 & 4.7 & Dorsolateral prefrontal (areas \\
\hline 33 & & & & 46 and 10$)$ \\
\hline 12 & -1 & 58 & 4.7 & SMA (area 6) \\
\hline & & & & Right hemisphere \\
\hline-42 & -49 & 40 & 7.4 & Posterior parietal (area 40) \\
\hline-30 & 3 & 47 & 6.9 & SMA (area 6) \\
\hline & 28 & 29 & 5.5 & Dorsolateral prefrontal (areas \\
\hline-35 & & & & 46 and 9) \\
\hline-12 & -64 & 50 & 4.6 & Superior parietal (area 7) \\
\hline
\end{tabular}

SMA, supplementary motor area.

previously memorized spatial positions during the search task. Post-experimental questionnaires revealed that the predominant strategy for monitoring these locations involved imagery of the three positions, a process known to activate occipital cortex. ${ }^{35}$ Thus, we may have subtracted out occipital activations in this study. In line with this reasoning, we did find occipital activations in a previous spatial memory study that utilized a simpler control condition. ${ }^{2}$

In summary, our study of spatial working memory shows a striking overlap in the brain regions that mediate spatial selective attention and spatial working memory. This provides support for the hypothesis that the brain regions subserving spatial attention may also mediate spatial memory.

\section{Dual-Task Memory Experiment}

\section{A Prediction}

In addition to predicting correspondence in the neural circuitry of spatial memory and attention, the proposed model of spatial working memory makes a behavioral prediction. If spatial rehearsal involves selective orienting to memorized locations, then the typical effect of orienting spatial selective attention-improved processing efficiency at attended locations-should be observable at memorized locations.

\section{Method}

To test this prediction, a dual-task spatial memory experiment was designed (illustrated in FIG. 9). Each trial consisted of the following sequence of events: (1) The appearance of a central fixation cross marked the beginning of each trial 


\section{Dual Task Memory Experiment}

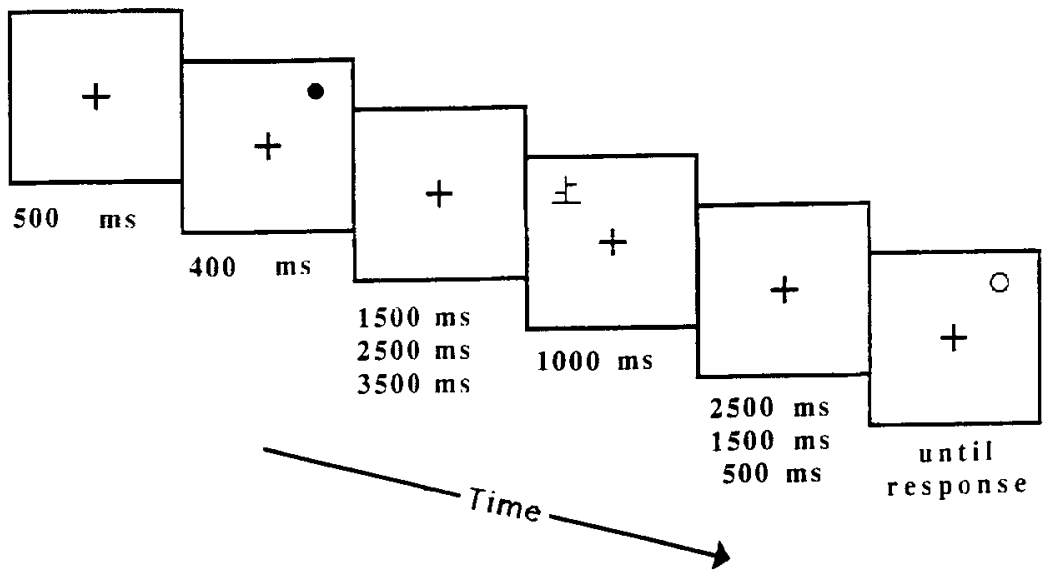

FIGURE 9. Schematic drawing of the sequence of events in each trial of the Dual-Task Spatial Memory experiment. A $50-\mathrm{ms}$ warning tone occurred at the onset of the fixation cross. Choice stimuli (middle panel) occurred at intervals varying randomly from 1500,2500 , and $3500 \mathrm{~ms}$ after the offset of the dot. The retention interval totaled $5000 \mathrm{~ms}$.

(a warning tone also occurred during the first $50 \mathrm{~ms}$ of this period). (2) The memory stimulus (a simple dot) was presented for $400 \mathrm{~ms}$ (the potential locations of the dot included 36 positions on each of three concentric circles, at $4,5.5$, and $7^{\circ}$ of visual angle; the dot fell on each circle and position with equal likelihood). (3) A 5000-ms retention interval ensued, during which a choice reaction time stimulus appeared for $1000 \mathrm{~ms}$ (potential locations were from the same set as the memorized locations). The choice stimulus occurred at an interval varying randomly between 1500,2500 , and $3500 \mathrm{~ms}$ after the offset of the memory stimulus; (4) $5000 \mathrm{~ms}$ after the offset of the dot, a probe circle appeared on the screen and remained visible until the subject indicated with a key press whether or not it encircled the original dot.

The probe matched the memorized location with probability .5 (hit trials). When the probe did not fall in the memorized location (miss trials), the distance between the probe and the dot was varied systematically between $.7,2,2.7$, and $3.7^{\circ}$ of visual angle. With probability 25 , the choice stimulus appeared in the same location as the one held in memory (constituting a "choice match"). In order to control for retinal acuity effects, choice matches and choice mismatches were equally distributed on the three circles.

Eight university students served as subjects. Subjects were instructed to regard the memory task as primary, and not to let the choice task interfere with memory accuracy. Subjects were instructed to maintain fixation throughout each trial, and they were monitored with a video camera in order to ensure compliance. Trials in which eye movements were detected were excluded. 
Results

Results on the memory task were orderly. Mean accuracy on hit trials was .87 . Accuracy on the miss trials increased monotonically as the distance of the probe from the memorized location increased. This distance effect shows that subjects were probably using a spatial coding strategy to perform the primary memory task.

The critical variable in this experiment is reaction time to the choice matches compared to choice mismatches. If subjects are selectively attending the memorized locations, there should be a corresponding decrease in reaction time to the choice matches. The results confirmed this prediction. Subjects were significantly faster to respond to choice matches than to choice mismatches (598 vs. $615 \mathrm{~ms}$ ), $[\mathrm{f}(1.7)=10.6, p<.025]$. Although accuracy on this task was virtually at ceiling, subjects were also reliably more accurate on choice match trials than choice mismatch trials $(99.3$ vs. $97.9 \%),[t(7)=2.1, p<.05$, one-tailed], eliminating the possibility of a speed/accuracy trade-off.

Although these results support the hypothesis that spatial rehearsal is mediated by orienting of spatial selective attention, they should be interpreted with caution. Further control experiments are needed to assess potential alternative explanations of these data. For instance, it is possible that the dot marking the memorized location caused automatic orienting of spatial attention to the memorized location (regardless of whether this orienting might subserve rehearsal). Although the interval between the memory stimulus and the choice stimulus (minimum $1500 \mathrm{~ms}$ ) exceeds previously demonstrated durations of exogenous orienting, ${ }^{36}$ this possibility should be considered. Also, it is possible that subjects adopted a voluntary orienting strategy (not in service of, but in addition to, the memory task). Although the memory dot matched the choice location only with probability .25 , it is still possible that subjects adopted the (nonoptimal) strategy of attending to the memorized location in order to facilitate performance on the choice task.

\section{Conclusions}

Preliminary behavioral evidence shows that when subjects hold a location in working memory, visual processing benefits attributable to spatial selective attention can be observed at that location. Electrophysiological, neuroimaging, and clinical evidence suggests a strong correspondence between the neurological substrates of spatial selective attention and spatial working memory. Thus, behavioral and brain evidence converge to suggest a role for spatial selective attention in the maintenance of information in spatial working memory.

The dissociation of storage and rehearsal processes is less clear in spatial working memory than it is in verbal working memory; however, the distinction between the source and the site of attentional effects ${ }^{37}$ may provide a useful perspective. That is, if the visual processing areas are regarded as the site of attentional effects, whereas frontal and superior parietal regions serve as the source of these effects, a pleasing analogy can be drawn between the site/source distinction, and the storage/ rehearsal distinction. By this view, the location-specific change in visual process- 
ing regions could constitute storage in spatial working memory, whereas the active maintenance of these changes by frontal and parietal cortices could constitute rehearsal.

\section{GENERAL CONCLUSIONS}

Consistent with our initial hypotheses, neuroanatomical and behavioral evidence shows that verbal working memory involves dissociable storage and rehearsal processes. Verbal storage recruits mechanisms in posterior brain regions, whereas anterior regions mediate articulatory rehearsal. In addition, we advanced a hypothesis in which spatial selective attention mediates the active maintenance of information in spatial working memory. This model is supported by both neuroanatomical and behavioral evidence. Here again, frontal brain regions may play an important role in rehearsal (along with other attentional mechanisms in parietal cortex), whereas posterior (visual processing) brain regions may be viewed as a passive buffer for location information.

\section{REFERENCES}

1. Goldman-Rakic, P. S. 1987. In Handbook of Physiology: The Nervous System. F. Plum, Ed. American Physiological Society. Bethesda, MD.

2. Jonides, J., E. E. Smith, R. A. Koeppe, E. Awh, S. Minoshima \& M. Mintun. 1993. Spatial working memory in humans as revealed by PET. Nature 363: 623-625.

3. Wilson, F. A. W. S. P. O'Scalaidue \& P. S. Goldman-Rakic. 1993. Dissociation of object and spatial processing domains in primate prefrontal cortex. Science 260: $1955-1958$.

4. Smith, E. E., J. Jonides, R. A. Koeppe, E. Awh, E. H. Schumacher \& S. Minoshima. Spatial vs. object working memory: PET investigations. J. Cognit. Neurosci. 7: $337-358$.

5. Sperling, G. 1967. Successive approximations to a model for short-term memory. Acta Psychologica 27: 285-292.

6. Baddel.EY, A. D. 1986. Working Memory. Clarendon Press. Oxford, UK.

7. Baddeley, A. D., N. Thompson \& M. BuChanan. 1975. Word length and the structure of short-term memory. J. Verb. Learn. Verb. Behav. 14: 575-589.

8. Awh, E., J. Jonides, E. E. Smith. E. H. Schumacher, R. A. Koeppe \& S. Katz. Dissociation of storage and rehearsal in verbal working memory: Evidence from PET. Psychol. Sci. In press.

9. Cowan, N. 1994. Mechanisms of verbal short-term memory. Curr. Directions Psychol. Sci. 3(6): 185-189.

10. Sternberg, S. 1966. High speed scanning in human memory. Science 153: 652-654.

11. Posner, M. I., S. E. Petersen, P. T. Fox \& M. E. Raichle. 1988. Localization of cognitive operations in the human brain. Science 240: 1627-1631.

12. Minoshima, S., K. L. Berger, K. S. Lee \& M. A. Mintun. 1992. An automated method for rotational correction and centering of three-dimensional functional brain images. J. Nucl. Med. 33: 1579-1585.

13. Minoshima, S., R. A. Koeppe, M. A. Mintun, K. L. Berger, S. F. Taylor, K. A. Frey \& D. E. Kuhl. 1993. Automated detection of the intercommissural line for stereotactic localization of functional brain images. J. Nucl. Med. 34: 322-329.

14. Talairach, J. \& P. Tournoux. 1988. A Co-Planar Stereotaxic Atlas of a Human Brain. Thieme. Stuttgart.

15. Fox, P. T., J. M. Fox, M. E. Raichle \& R. M. Burde, 1985. The role of cerebral 
cortex in the generation of saccadic eye movements; a positron emission tomography study. J. Neurophysiol. 54: 348-368.

16. Friston, K. J., C. D. Frith, P. F. Liddle \& R. S. J. Frackowiak. 1991. Comparing functional (PET) images: The assessment of significant change. J. Cereb. Blood Flow Metab. 11: 690-699.

17. Worsley, J. J., A. C. Evans, S. Marrett \& P. Neelin. 1992. A three-dimensional statistical analysis for CBF activation studies in the human brain. J. Cereb. Blood Flow Metab. 12: 900-918.

18. Petersen, S. E.. P. T. Fox, M. I. Posner, M. Mintun \& M. E. Raichle. 1988. Positron emission tomographic studies of the cortical anatomy of single-word processing. Nature 331: 585-589.

19. MCCARThY, R. A. \& E. K. WarRington. 1990. In Cognitive Neuropsychology: A Clinical Introduction. Academic Press. San Diego. CA.

20. Vallar, G. \& T. Shallice. 1990. Neuropsychological Impairments of Short Term Memory. Cambridge University Press. Cambridge, UK.

21. Paulesu, E., C. D. Frith \& R. S. J. Frackowiak. 1993. The neural correlates of the verbal component of working memory. Nature 362: 342-343.

22. PARdo, J. V., P. J. PARdo, K. W. Janer \& M. E. Raichle. 1990. The anterior cingulate cortex mediates processing selection in the Stroop attentional conflict paradigm. Proc. Natl. Acad. Sci. USA 87: 256-259.

23. Fuster, J. M. 1995. Memory in the Cerebral Cortex. MIT Press. Cambridge, MA.

24. Roland, P. E. \& L. Friberg. 1985. Localization of cortical areas activated by thinking. J. Neurophysiol. 53: 1219-1243.

25. Petrides, M., B. Alivisatos, E. Meyfer \& A. Evans. 1993. Functional activation of the human frontal cortex during the performance of verbal working memory tasks. Proc. Natl. Acad. Sci. USA 90: 878-882.

26. SMith, E. E., J. Jonides \& R. A. Konppe. Dissociating verbal and spatial working memory using PET. Cereb. Cortex. In press.

27. SMYTh, M. M. \& K. A. SCHOLEY. 1994. Interference in immediate spatial memory. Memory \& Cognition 22(1): $1-13$.

28. Posner, M. I. 1980. Orienting of attention. Q. J. Exp. Psychol. 32: 3-25.

29. Heinze, H. J., G. R. Mangun, W. Burchert, H. Hinrichs, M. Scholz, T. F. Munte, A. Gos, M. Scherg, S. Johannes, H. Hundeshagen, M. S. Gazzaniga \& S. A. Hiliyard. 1994. Combined spatial and temporal imaging of brain activity during visual selective attention in humans. Nature 372: 543-546.

30. Petersen, S. E., M. Corbetta, F. M. Miezin \& G. L. Shulman. 1994. Pet studies of parietal involvement in spatial attention: Comparison of different task types. Can. J. Exp. Psychol. 48(2): 319-338.

31. Kodaka, Y., A. Mikami \& K. Kubota. 1994. Aitention to a visual stimulus enhances neuronal responses in monkey prefrontal cortex (poster). Presented at the Society of Neuroscience meeting. Washington. D.C.

32. Petrides, M. \& D. N. PANDYA. 1994. Comparative architectonic analysis of the human and the macaque frontal cortex. In F. Boller \& J. Grafman. Eds.: 17-57. Handbook of Neuropsychology, Vol. 9. Elsevier. Amsterdam.

33. Foster, J. K., G. A. Eskes \& D. T. Stuss. 1994. The cognitive neuropsychology of attention: A frontal lobe perspective. Spec. Issue: The cognitive neuropsychology of attention. Cogn. Neuropsychol. 11(2): 13.3-147.

34. Alivisatos, B. \& B. Milner. 1989. Effects of frontal or temporal lobectomy on the use of advance information in a choice reaction time task. Neuropsychologia 27: $495-503$.

35. Kosslyn, S. M., N. M. At.pert, W. L. Thompson, V. Malijkovic, S. Weisf, C. F. Chabris, S. E. Hamil.ton, S. L. Rauch \& F. S. Buonanno. 1993. Visual mental imagery activates topographically organized visual cortex: PET investigations. J. Cognit. Neurosci. 5(3): 263-287.

36. Mueller, H. J. \& P. M. RabBitT, 1989. Reflexive and voluntary orienting of visual attention: Time course of activation and resistance to interruption. J. Exp. Psychol. Hum. Percept. Perform. 15(2): 315 -330.

37. Posner, M. I. \& S. E. Petersen. 1990. The attention system of the human brain. Annu. Rev. Neurosci. 13: 25-42. 